\title{
On the treatment of uncertainty in experimentally measured frequency response functions
}

\author{
J.W.R. Meggitt ${ }^{1}$ \\ ${ }^{1}$ Acoustics Research Centre, University of Salford, Greater Manchester, M5 4WT
}

\begin{abstract}
Frequency response functions (FRFs) are routinely used within experimental structural dynamics, among other fields. The uncertainty associated with their measurement is typically modelled on the simplified assumption that additive uncorrelated noise is present. This treatment of FRF uncertainty is questioned here by demonstrating that the notion of operator uncertainty (i.e. the uncertainty due to inconsistent force excitation) introduces a correlation between measured FRFs which is not accounted for under the assumption of additive noise. The origin of this correlation is illustrated and its experimental presence verified. The importance of this correlation on the propagation of uncertainty is subsequently investigated as part of a numerical and experimental case study, where its influence on the inversion of an uncertain FRF matrix is considered. It is shown that the neglect of such correlations can lead to large errors in uncertainty estimates, for example in dynamic sub-structuring and the solution of inverse problems.
\end{abstract}

Keywords: Frequency response function, structural dynamics, experimental, measurement, operator uncertainty

\section{Introduction}

The measurement of frequency response functions (FRFs, a frequency domain representation of a system's dynamic characteristics) is routine within experimental structural dynamics, among other fields, and is often the first step in some further analysis, for example, modal parameter extraction [1], system identification [2], dynamic sub-structuring [3] or inverse force identification [4]. Often these measured FRFs are used (perhaps as part of one or more of the above procedures) to predict and/or analyse the dynamics of complex built-up structures. In many cases, for example, aerospace and automotive applications, these structures are designed so as to conform to strict limits, be it to avoid structural fatigue and failure, or to promote passenger comfort. To this end, FRF based analysis is an essential step is fulfilling the above. That said, as with any measured quantity, FRFs are subject to a degree of uncertainty, a proper understanding of which is essential if limits are to be met with confidence.

FRFs are used extensively in both academic and industrial settings and form the basis of numerous analyses. Their importance is recognised in [5] where an ISO standardised measurement procedure is given. However, there remain many unresolved issues relating to standardisation, e.g. a lack of accepted rules on how to present complex narrow band data (various similar but different formats exist and are in common use to describe essentially the same thing: power spectral density, Fourier spectrum, power spectrum, etc.). Further issues arise regarding frequency resolution and measurement units. In this paper we will address some unresolved issues related to the uncertainties present in measured FRFs.

Whilst the measurement of structural FRFs is an ISO standardised procedure, minimal guidance is given regarding the treatment of their uncertainty. In [5] it is suggested that co- herence be used to access the confidence in repeated measurements, alongside repeatability, reciprocity and linearity checks. This does not, however, provide an adequate description of their uncertainty such that it may be propagated when the measured FRFs are subject to further analysis.

This paper will discuss the necessary requirements for an appropriate treatment of uncertainty in complex measured FRFs. In doing so we will discuss the notion of operator uncertainty and the inter-FRF correlation that it introduces. The importance of this correlation in the subsequent propagation of uncertainty will also be considered.

When estimating the uncertainty of measured FRFs one must consider the experimental procedure undertaken [6], i.e. Single Input/Single Output (SISO), Single Input/Multiple Output (SIMO) or Multiple Input/Multiple Output (MIMO), as each of the above will require a different treatment of uncertainty. As an example, consider the SISO approach where a number of (individual) FRFs are measured in turn. Under the assumption that the measurement of each FRF is statistically independent, there is unlikely to exist any correlation between the measured FRFs. However, if one were to measure multiple FRFs simultaneously, for example, through a SIMO approach, there would exist the possibility of correlation between measured FRFs, since they are acquired as part of the same measurement. The term correlation here is meant generally in that it refers to an arbitrary statistical dependence between two measured FRFs. Whilst the nature of this correlation will depend upon the underlying cause of uncertainty, it may be interpreted as a frequency dependent tendency for the real and imaginary components of one or more FRFs to deviate from their expected values in a similar way.

The notion of inter-FRF correlation is of particular importance in experimental structural dynamics, where SIMO methods are routine (i.e. roving hammer excitation with multiple 
response sensors). The standard treatment of FRF uncertainty, however, is to assume an additive noise on the system outputs, typically of a Gaussian form, and neglect the effect of any such correlations. Whilst in the presence of a low level excitation one might expect uncorrelated uncertainty (e.g. measurement noise) to dominate, for a desirable signal to noise ratio (SNR) correlated uncertainty (e.g. operator) is more likely to dominate, as it is largely independent of excitation level. As such, the assumption of uncorrelated uncertainty is unlikely to be valid in most experimental scenarios.

In this paper we will introduce the notion of operator uncertainty (also referred to as measurement bias in [7, 8]), which describes the uncertainty introduced as a result of human error in the measurement procedure. Operator uncertainty applies in particular to impact based FRF measurements, where it describes the underlying uncertainty in the applied excitation. This uncertainty is introduced as a result of an inconsistent excitation position, and/or orientation, when repeated measurements are made. Although we are concerned primarily with operator uncertainty as a source of correlation, external factors may introduce additional correlations if their influence is registered by multiple sensors simultaneously, for example, imperfect boundary conditions, changes in temperature, etc.

Whilst impact excitation is preferred by many (as opposed to shaker excitation) it comes with an increased uncertainty in the position of excitation, especially if access is limited and conditions are unfavourable. This is of particular importance in dynamic sub-structuring (DSS), modal analysis, and transfer path analysis, where it has been shown that inconsistencies in measured FRF matrices can lead to: the extraction of incorrect modal parameters [9], spurious peaks in sub-structured FRF predictions [10], and large errors in reconstructed force estimates [11]. This further highlights the need for an appropriate treatment of FRF uncertainty in experimental structural dynamics.

The early work of Bendat et al. [12, 6, 13] sees the formulation of the now common statistical error analysis relations used in the quantification of uncertainty in measured input/output relations, such as coherence and FRFs. These relations, which are based on the assumption of small and uncorrelated uncertainty, have been used by many, particularly in the field of structural health monitoring $[14,15]$, to establish confidence bounds on measured FRFs that are only exceeded in the presence of damage.

A more general treatment of FRF uncertainty was presented in [16] where the authors adopted a bivariate description of the complex uncertainty associated with measured FRFs. The effect of calibration, cosine, and mass loading uncertainty were considered and applied to the analysis of 100 repeated FRF measurements on a tool-holder-spindle-machine assembly. Although correlation between the real and imaginary components of the FRF was included, analysis was limited to a single point FRF. As such, the effect of operator uncertainty and inter-FRF correlation was not considered.

The bivariate description of complex uncertainty, and its propagation, was previously presented in [17] where the authors were concerned with the estimation of complex reflection and transmission coefficients (i.e. s-parameters) encountered in radio frequency and microwave science. This approach was further elaborated in [18], with an emphasis on the propagation of complex uncertainty.

In [19] Schultz et al. derive expressions for the uncertainty in FRF estimates when using a periodic random input for two system models (output noise only, and uncorrelated input and output noise), and further consider the propagation of uncertainty from real and imaginary components onto magnitude and phase descriptors. Whilst the authors acknowledge the correlation between real and imaginary components of an FRF, they considered only SISO estimation, and therefore neglect the effect of operator uncertainty.

Interested in the effect of FRF uncertainty on DSS procedures, de Klerk and Visser [20] investigated the experimental error associated with inconsistent excitation and response orientation (referred to here as operator uncertainty), particularly in the measurement of point FRFs. In [7] de Klerk went on to derive an expression for the variance of a coupled FRF from a sub-structuring prediction, based on the law of error propagation and the uncertainty of the uncoupled sub-structure FRFs. It was assumed that the uncertainties present in the uncoupled FRFs were small, obeyed a Gaussian distribution and, importantly, were uncorrelated. In [8] Voormeeren et al. further develop de Klerk's propagation framework [7]. The underlying assumptions (small uncorrelated uncertainty) were retained, although the authors did acknowledge its limitation, noting that, 'When the noise on the signals is 'mechanical' of nature (e.g., vibrations from the environment, fluctuations in applied excitation, etc.), the errors on the measured signals will be highly correlated due to the physical structure'. Furthermore, whilst the authors considered the separation of real and imaginary components, it appears they chose not to account for their possible correlation, nor the effect of inter-FRF correlation.

Using a bivariate description, Mao and Todd [21] attempted to quantify the uncertainty in the magnitude and phase of an FRF using a random excitation and the $\mathrm{H} 1$ estimator. The authors make the simplifying assumption that the real and imaginary components of an FRF have the same variance, and that they are uncorrelated. Since a SISO estimation was considered, inter-FRF correlation was not.

Concerned with the propagation of uncertainty through inverse problems, such as force identification, Meggitt et al. [22] proposed a general framework where the uncertainty of a measured FRF matrix was characterised using the bivariate description. Whilst the authors considered the FRF uncertainty generally such that there could exist any number of correlations, they did not investigate whether or not such correlations existed, nor their influence on the propagation of uncertainty. Nevertheless, the authors went on to investigate the propagation of uncertainty through a matrix inversion and derived a linearised relation for doing so. The inversion of measured FRF matrices is an operation of practical importance in both DSS procedures and in the solution of inverse problems.

Whilst the above literature illustrates an interest in the uncertainty of measured FRFs, with the exception of [22], there are few works, if any, that consider more generally its underly- 
ing nature. It is typically assumed that the uncertainties present in measured FRFs are independent and identically distributed (i.i.d). Whilst the latter may be fair, the assumption of independence is likely invalid if the FRFs are measured simultaneously through some shared excitation (for example using SIMO or MIMO procedures). In such a case, it is hypothesised that a correlation is introduced through the shared underlying (operator) uncertainty, and that this correlation is scaled according to the dynamics of the assembly. With that said, it is the primary aim of this paper is to illustrate that such a correlation is introduced between measured FRFs when the notion of operator uncertainty (i.e the influence of a random excitation position) is considered. A further aim is to investigate the extent to which, if any, this inter-FRF correlation influences the propagation of uncertainty. Of particular interest is the influence of inter-FRF correlation on the propagation of uncertainty through matrix inversions, which are essential in both DSS procedures and in the solution to inverse problems.

The remainder of this paper will be organised as follows. Section 2 will begin by deriving some statistical relations for 'measured' FRFs subject to operator uncertainty. These relations will be corroborated against experimental data in section 2.4. The consequence of inter-FRF correlation on the propagation of uncertainty will be investigated in section 3 as part of a numerical study. Lastly, section 5 will draw some concluding remarks.

\section{Theory}

Before proceeding with the analysis of 'measured' (or uncertain) FRFs, it is useful to first categorise the types of uncertainty that one may encounter. For passive structural properties, such as FRFs, Meggitt et al. [22] categorise experimental uncertainty as either measurement or operator based. Measurement uncertainty describes the cumulative effect of noise sources within the measurement signal path and beyond, and includes, for example: external disturbances, thermo-electrical noise, sampling error, finite precision, etc. Measurement uncertainty may be considered aleatory in that it is largely unavoidable, but may be reduced to some extent. Operator uncertainty describes the effect of human error in the measurement procedure, for example, inconsistent location and/or orientation of excitation forces. Operator uncertainty may be considered epistemic as it may be reduced by carefully executed experimentation.

As discussed in section 1, previous works have considered almost exclusively the influence of measurement uncertainty on measured FRFs. ${ }^{1}$ This is typically modelled by considering a measured FRF in the form,

$$
H_{b a}^{\text {meas }}=H_{b a}^{\text {true }}+\epsilon
$$

where $\epsilon$ is a noise term distributed according to a complex normal distribution whose real and imaginary components may or

\footnotetext{
${ }^{1}$ Measurement uncertainty is also a common consideration in other parameter estimations, for example, inverse problems of the form $a=X b+\epsilon$ (e.g. force reconstruction [23, 24, 25]).
}

may not be correlated. Whilst the above may be valid in the presence of measurement uncertainty, it is likely not sufficient for describing the effect of operator uncertainty.

To investigate the nature of operator uncertainty a measured FRF may be considered an output of an FRF function of the random variable (RV) $a=a_{0}+\mathbf{a}$, where $\mathbf{a}$ is a RV (given in bold to distinguish from other non-random variables) which describes the random variation around the intended excitation position $a_{0}$.

$$
H_{b a}^{\text {meas }}=H_{b a}^{\text {true }}\left(a_{0}+\mathbf{a}\right)
$$

In what follows we will consider this more general treatment of experimental uncertainty. In the following analysis, the uncertainty of measured FRFs will be investigated by considering a modal summation whose excitation position is considered a RV. Firstly, however, a brief moment will be taken to discuss the treatment of complex FRF uncertainty using the bivariate description.

\subsection{On the Treatment of Complex Uncertainty}

The FRFs typically encountered in experimental structural dynamics are complex quantities, acquired through the ratio of Fourier transformed input and output signals [6]. Typical examples include accelerance, mobility and receptance, which are given as the ratio of acceleration, velocity and displacement, respectively, to an applied force [26].

The statistical properties of a complex RV, $H \in \mathbb{C}$, such as an FRF, may be described generally by the complex bivariate variance-covariance matrix $[17,18,16,19,21]$,

$$
\boldsymbol{\Sigma}_{H}=\left[\begin{array}{ll}
\sigma_{\mathfrak{R}(H) \mathfrak{R}(H)} & \sigma_{\mathfrak{R}(H) \mathfrak{I}(H)} \\
\sigma_{\mathfrak{I}(H) \mathfrak{R}(H)} & \sigma_{\mathfrak{I}(H) \mathfrak{I}(H)}
\end{array}\right]
$$

where, $\sigma_{\mathfrak{R}(H) \mathfrak{R}(H)}$ is the variance of the real part of $H, \sigma_{\mathfrak{I}(H) \mathfrak{I}(H)}$ is the variance of the imaginary part of $H$, and $\sigma_{\mathfrak{I}(H) \mathfrak{R}(H)}=$ $\sigma_{\mathfrak{R}(H) \mathfrak{I}(H)}$ is the covariance between them. This notion may readily be extended to describe the covariance between complex RVs,

$$
\boldsymbol{\Sigma}_{H_{1} H_{2}}=\left[\begin{array}{cc}
\sigma_{\mathfrak{R}\left(H_{1}\right) \mathfrak{R}\left(H_{2}\right)} & \sigma_{\mathfrak{R}\left(H_{1}\right) \mathfrak{I}\left(H_{2}\right)} \\
\sigma_{\mathfrak{I}\left(H_{1}\right) \mathfrak{R}\left(H_{2}\right)} & \sigma_{\mathfrak{I}\left(H_{1}\right) \mathfrak{I}\left(H_{2}\right)}
\end{array}\right] .
$$

Consequently, the uncertainty of a measured FRF matrix $\mathbf{H} \in$ $\mathbb{C}^{M \times N}$ is completely described by the variance covariance matrix $\Sigma_{\mathbf{H}} \in \mathbb{R}^{2 M N \times 2 M N}$, where $M$ and $N$ represent, respectively, the number of response and excitation positions considered. This may be obtained experimentally through the repeated excitation of each measurement position (see Appendix A).

Whilst the above provide a general description of complex uncertainty, in the FRF literature it is often assumed that $\boldsymbol{\Sigma}_{H}=$ $\operatorname{diag}\left(\sigma_{\mathfrak{R}(H) \mathfrak{R}(H)}, \sigma_{\mathfrak{I}(H) \mathfrak{I}(H)}\right)$ and that $\boldsymbol{\Sigma}_{H_{1} H_{2}}=\mathbf{0}$. That is, the uncertainties are uncorrelated. Although this assumption simplifies the treatment of uncertainty greatly, its validity is questionable, particularly if one considers the effect of operator uncertainty, as will be shown in the following section.

\subsection{First Order Statistical Properties - Expectation}

Consider the receptance FRF of an arbitrary, proportionally damped, structure in the form of a modal summation [1],

$$
H_{b a}(\omega)=\sum_{r}^{N} \frac{\Phi_{b r}(b) \Phi_{a r}\left(a_{0}+\mathbf{a}\right)}{\omega_{r}^{2}-\omega^{2}+i \omega_{r} \eta_{r}}
$$


where, $\omega$ is the frequency at which the FRF is evaluated, $\Phi_{b r}(b) \in$ $\mathbb{R}$ is the response mode shape function of the positional argument $b, \Phi_{a r}\left(a_{0}+\mathbf{a}\right) \in \mathbb{R}$ is the excitation mode shape function of the positional argument $a=a_{0}+\mathbf{a}$, where $a_{0}$ represents the intended excitation position and a a RV (given in bold to distinguish from other non-random variables) embodying the operator uncertainty, $\omega_{r}$ is the $r$ th natural frequency, and $\eta_{r}$ is its associated loss factor. Equation 5 describes generally a measured FRF subject to operator uncertainty only. The additional influence of measurement uncertainty $\epsilon$ is not considered here.

We are not only interested in demonstrating the statistical relation between the entries of an FRF matrix, but also their real and imaginary components. As such, we separate the above summation as so,

$$
\begin{aligned}
H_{b a}=\sum_{r}^{N}\left[\frac{\left(\omega_{r}^{2}-\omega^{2}\right) \Phi_{b r}(b) \Phi_{a r}\left(a_{0}+\mathbf{a}\right)}{\left(\omega_{r}^{2}-\omega^{2}\right)^{2}+\left(\omega_{r} \eta_{r}\right)^{2}}-\right. & \\
& \left.i \frac{\omega_{r} \eta_{r} \Phi_{b r}(b) \Phi_{a r}\left(a_{0}+\mathbf{a}\right)}{\left(\omega_{r}^{2}-\omega^{2}\right)^{2}+\left(\omega_{r} \eta_{r}\right)^{2}}\right] .
\end{aligned}
$$

The expectation of the real part $\mathfrak{R}\left(H_{b a}\right)$,

$$
\mathbb{E}\left[\mathfrak{R}\left(H_{b a}\right)\right]=\mathbb{E}\left[\sum_{r}^{N} \frac{\left(\omega_{r}^{2}-\omega^{2}\right) \Phi_{b r}(b)}{\left(\omega_{r}^{2}-\omega^{2}\right)^{2}+\left(\omega_{r} \eta_{r}\right)^{2}} \Phi_{r}\left(a_{0}+\mathbf{a}\right)\right]
$$

is readily found by noting the linearity of the expectation operator, $\mathbb{E}[X+c Y]=\mathbb{E}[X]+\mathbb{E}[c Y]$, where $X$ and $Y$ represent arbitrary RVs and $c$ a constant. Since $\Phi_{r}\left(a_{0}+\mathbf{a}\right)$ is the only $\mathrm{RV}$ present, the remaining quotient is a constant and the above reduces to,

$$
\mathbb{E}\left[\mathfrak{R}\left(H_{b a}\right)\right]=\sum_{r}^{N} \frac{\left(\omega_{r}^{2}-\omega^{2}\right) \Phi_{b r}(b)}{\left(\omega_{r}^{2}-\omega^{2}\right)^{2}+\left(\omega_{r} \eta_{r}\right)^{2}} \mathbb{E}\left[\Phi_{r}\left(a_{0}+\mathbf{a}\right)\right]
$$

If we permit ourselves the assumption that the experimenter is well trained, the operator uncertainty is likely to be small (i.e the random deviation a will have a low variance), then $\Phi_{a r}\left(a_{0}+\right.$ a) may be expanded as a first order Taylor series,

$$
\begin{aligned}
& \mathbb{E}\left[\mathfrak{R}\left(H_{b a}\right)\right]=\sum_{r}^{N} \frac{\left(\omega_{r}^{2}-\omega^{2}\right) \Phi_{b r}(b)}{\left(\omega_{r}^{2}-\omega^{2}\right)^{2}+\left(\omega_{r} \eta_{r}\right)^{2}} \\
&\left(\mathbb{E}\left[\Phi_{a r}\left(a_{0}\right)\right]+\mathbb{E}\left[\mathbf{a}\left(\frac{d \Phi_{a r}(a)}{d a}\right)_{a=a_{0}}\right]\right)
\end{aligned}
$$

where the derivative is taken with respect to excitation position, $a$, and is evaluated at the intended position $a_{0}$. Noting linearity, the above leads to,

$$
\begin{aligned}
\mathbb{E}\left[\mathfrak{R}\left(H_{b a}\right)\right]= & \sum_{r}^{N} \frac{\left(\omega_{r}^{2}-\omega^{2}\right) \Phi_{b r}(b)}{\left(\omega_{r}^{2}-\omega^{2}\right)^{2}+\left(\omega_{r} \eta_{r}\right)^{2}} \\
& \left(\Phi_{a r}\left(a_{0}\right)+\mathbb{E}[\mathbf{a}]\left(\frac{d \Phi_{a r}(a)}{d a}\right)_{a=a_{0}}\right) .
\end{aligned}
$$

If we allow ourselves a further assumption that the well trained experimenter is consistent and on average hits the intended location $a_{0}$, the operator uncertainty a will have a zero mean.
Consequently, the expected value of the real part of a measured FRF subject to operator uncertainty is given by,

$$
\mathbb{E}\left[\mathfrak{R}\left(H_{b a}\right)\right]=\sum_{r}^{N} \frac{\left(\omega_{r}^{2}-\omega^{2}\right) \Phi_{b r}(b) \Phi_{a r}\left(a_{0}\right)}{\left(\omega_{r}^{2}-\omega^{2}\right)^{2}+\left(\omega_{r} \eta_{r}\right)^{2}} .
$$

Similarly, the expected value of the imaginary component is given by,

$$
\mathbb{E}\left[\mathfrak{J}\left(H_{b a}\right)\right]=-\sum_{r}^{N} \frac{\omega_{r} \eta_{r} \Phi_{b r}(b) \Phi_{a r}\left(a_{0}\right)}{\left(\omega_{r}^{2}-\omega^{2}\right)^{2}+\left(\omega_{r} \eta_{r}\right)^{2}} .
$$

Equation 11 and 12 represent the underlying true FRF of the structure. The above illustrates that, provided the assumption of zero mean and small variance are met, no bias is introduced as a result of operator uncertainty. Note that the inclusion of measurement uncertainty would yield the same expectations, since $\mathbb{E}[\epsilon]=\mathbf{0}$.

\subsection{Second Order Statistical Properties - Covariance}

Having derived the expectation of the real and imaginary components, we will now consider the second order statistical relationships between the real and imaginary components of two measured FRFs, $H_{b a}$ and $H_{c a}$, who share the excitation position $a$.

We will begin by considering their real parts. The covariance between $\mathfrak{R}\left(H_{b a}\right)$ and $\mathfrak{R}\left(H_{c a}\right)$ is defined as,

$$
\begin{aligned}
& \operatorname{Cov}\left[\mathfrak{R}\left(H_{b a}\right), \mathfrak{R}\left(H_{c a}\right)\right]=\mathbb{E}[\left(\mathfrak{R}\left(H_{b a}\right)-\mathbb{E}\left[\mathfrak{R}\left(H_{b a}\right)\right]\right) \\
&\left.\left(\mathfrak{R}\left(H_{c a}\right)-\mathbb{E}\left[\mathfrak{R}\left(H_{c a}\right)\right]\right)\right] .
\end{aligned}
$$

Substituting for the real part, and its expectation derived in section 2.2, this becomes,

$$
\begin{gathered}
\operatorname{Cov}\left[\mathfrak{R}\left(H_{b a}\right), \mathfrak{R}\left(H_{c a}\right)\right]=\mathbb{E}\left[\left(\sum_{r}^{N} \frac{\left(\omega_{r}^{2}-\omega^{2}\right) \Phi_{b r}(b) \Phi_{r}\left(a_{0}+\mathbf{a}\right)}{\left(\omega_{r}^{2}-\omega^{2}\right)^{2}+\left(\omega_{r} \eta_{r}\right)^{2}}\right.\right. \\
\left.-\sum_{r}^{N} \frac{\left(\omega_{r}^{2}-\omega^{2}\right) \Phi_{b r}(b) \Phi_{a r}\left(a_{0}\right)}{\left(\omega_{r}^{2}-\omega^{2}\right)^{2}+\left(\omega_{r} \eta_{r}\right)^{2}}\right)\left(\sum_{r}^{N} \frac{\left(\omega_{r}^{2}-\omega^{2}\right) \Phi_{c r}(b) \Phi_{r}\left(a_{0}+\mathbf{a}\right)}{\left(\omega_{r}^{2}-\omega^{2}\right)^{2}+\left(\omega_{r} \eta_{r}\right)^{2}}\right. \\
\left.\left.-\sum_{r}^{N} \frac{\left(\omega_{r}^{2}-\omega^{2}\right) \Phi_{c r}(b) \Phi_{a r}\left(a_{0}\right)}{\left(\omega_{r}^{2}-\omega^{2}\right)^{2}+\left(\omega_{r} \eta_{r}\right)^{2}}\right)\right]
\end{gathered}
$$

If, for simplicity, we assume statistical independence between the modes, the above reduces to,

$$
\begin{aligned}
& \operatorname{Cov}\left[\mathfrak{R}\left(H_{b a}\right), \mathfrak{R}\left(H_{c a}\right)\right]= \sum_{r}^{N} \mathbb{E}\left[\left(\frac{\left(\omega_{r}^{2}-\omega^{2}\right) \Phi_{b r}(b) \Phi_{r}\left(a_{0}+\mathbf{a}\right)}{\left(\omega_{r}^{2}-\omega^{2}\right)^{2}+\left(\omega_{r} \eta_{r}\right)^{2}}\right.\right. \\
&\left.-\frac{\left(\omega_{r}^{2}-\omega^{2}\right) \Phi_{b r}(b) \Phi_{a r}\left(a_{0}\right)}{\left(\omega_{r}^{2}-\omega^{2}\right)^{2}+\left(\omega_{r} \eta_{r}\right)^{2}}\right)\left(\frac{\left(\omega_{r}^{2}-\omega^{2}\right) \Phi_{c r}(b) \Phi_{a r}\left(a_{0}+\mathbf{a}\right)}{\left(\omega_{r}^{2}-\omega^{2}\right)^{2}+\left(\omega_{r} \eta_{r}\right)^{2}}\right. \\
&\left.\left.-\frac{\left(\omega_{r}^{2}-\omega^{2}\right) \Phi_{c r}(b) \Phi_{a r}\left(a_{0}\right)}{\left(\omega_{r}^{2}-\omega^{2}\right)^{2}+\left(\omega_{r} \eta_{r}\right)^{2}}\right)\right]
\end{aligned}
$$

The assumption of independence between the modal contributions is to say that the each mode responds independently to a shared excitation, which is likely satisfied for well separated, 
uncoupled modes. In the case that the above assumption is not valid, a series of covariance terms must be included to account for the correlation between modes. For simplicity, however, we will assume the above assumption is valid.

After factorising common terms and utilising the definition,

$$
\alpha_{n r}=\frac{\left(\omega_{r}^{2}-\omega^{2}\right) \Phi_{n r}(b)}{\left(\omega_{r}^{2}-\omega^{2}\right)^{2}+\left(\omega_{r} \eta_{r}\right)^{2}}
$$

the above reduces to,

$$
\operatorname{Cov}\left[\mathfrak{R}\left(H_{b a}\right), \mathfrak{R}\left(H_{c a}\right)\right]=\sum_{r}^{N} \alpha_{b r} \alpha_{c r} \mathbb{E}\left[\left(\Phi_{r}\left(a_{0}+\mathbf{a}\right)-\Phi_{a r}\left(a_{0}\right)\right)^{2}\right]
$$

Noting that $\Phi_{a r}\left(a_{0}\right)=\mathbb{E}\left[\Phi_{r}\left(a_{0}+\mathbf{a}\right)\right]$, we arrive at,

$$
\operatorname{Cov}\left[\mathfrak{R}\left(H_{b a}\right), \mathfrak{R}\left(H_{c a}\right)\right]=\sum_{r}^{N} \alpha_{b r} \alpha_{c r} \operatorname{Var}\left[\Phi_{r}\left(a_{0}+\mathbf{a}\right)\right]
$$

Equation 18 states that the covariance between the real parts of two FRFs, $H_{b a}$ and $H_{c a}$, measured through a shared excitation, is given by summing the excitation mode shape variance over all modes, each scaled by an appropriate pair of coefficients, $\alpha_{n r}$.

Following a similar analysis the remaining covariances are found as,

$$
\operatorname{Cov}\left[\mathfrak{J}\left(H_{b a}\right), \mathfrak{J}\left(H_{c a}\right)\right]=\sum_{r}^{N} \beta_{b r} \beta_{c r} \operatorname{Var}\left[\Phi_{a r}\left(a_{0}+\mathbf{a}\right)\right]
$$

and

$$
\operatorname{Cov}\left[\mathfrak{R}\left(H_{b a}\right), \mathfrak{J}\left(H_{c a}\right)\right]=\sum_{r}^{N} \alpha_{b r} \beta_{c r} \operatorname{Var}\left[\Phi_{a r}\left(a_{0}+\mathbf{a}\right)\right]
$$

where a second coefficient $\beta_{n r}$ is defined,

$$
\beta_{n r}=\frac{\omega_{r} \eta_{r} \Phi_{n r}(b)}{\left(\omega_{r}^{2}-\omega^{2}\right)^{2}+\left(\omega_{r} \eta_{r}\right)^{2}} .
$$

Equation 18-20 describe the statistical relations between measured FRFs subject to operator uncertainty. If the influence of measurement uncertainty is also considered, the resulting FRF covariances are given simply as the sum of the operator and measurement based uncertainty contributions, since they are uncorrelated. Note however, that the measurement uncertainty will be non-zero only for those covariances that relate the real and imaginary components of the same FRF, i.e. for $b=c$.

In any case, other than a perfectly repeated excitation, the above return a finite non-zero covariance. The magnitude of this covariance is clearly proportional to the underlying operator uncertainty through the variance term, $\operatorname{Var}\left[\Phi_{a r}\left(a_{0}+\mathbf{a}\right)\right]$. The coefficient pair will amplify or attenuate this variance according to the dynamics of the assembly, as suggested in [8]. At resonance it can be seen that the uncertainty in the real part of the dominant mode vanishes, since $\alpha_{n r} \rightarrow 0$, leaving only the contribution of the remaining modes. Hence, at resonance the uncertainty in the real part is a minimum. Conversely, the imaginary part reaches a maximum at resonance, since $\beta_{n r} \rightarrow \frac{\Phi_{n r}(b)}{\omega_{r} \eta_{r}}$.
This is in agreement with experimental observations, as will be illustrated in section 2.4.

The above analysis clearly illustrates that when one takes into account the uncertainty associated with human error in the measurement procedure (which is referred to here as operator uncertainty) a correlation exists between the real and imaginary components of the measured FRFs. This is in opposition to what is commonly assumed and considered when investigating FRF uncertainty and its influence on any eventual parameter estimation.

Note that, if one considers the covariance between two FRFs that do not share an excitation, say $H_{b a}$ and $H_{b d}$, under the assumption that the underlying operator uncertainties are uncorrelated, $\operatorname{Cov}[\mathbf{a}, \mathbf{d}]=0$, which is a fair assumption as they correspond to different measurements, there will exist no correlation between them, and their covariances will be 0 . If, however, $H_{b a}$ and $H_{b d}$ were to be measured using an MIMO approach, then there may exist a correlation between them. The nature of uncertainty in MIMO estimations is considered beyond the scope of this work. Interest will instead focus on the SIMO procedure, which is most commonly used.

\subsection{Experimental Evidence}

In this section we present the results of a simple experiment that not only confirms the existence of operator uncertainty but demonstrates its correlated nature.

A freely suspended beam was instrumented with two accelerometers, positioned roughly $15 \mathrm{~cm}$ apart. An excitation was performed at one of their positions. This excitation was repeated 20 times and the resultant mobility FRFs (denoted $Y$ ) were calculated. The magnitudes of each point (excitation and response at the same location) and transfer (excitation and response at different locations) FRF are shown in figure 1a, in black and grey, respectively.

With the aim of first illustrating that there exists a correlation between the real and imaginary components of a measured FRF, the point and transfer FRFs of figure 1a are shown on Nyquist plots in figures $1 \mathrm{~b}$ and $1 \mathrm{c}$, respectively. The measured FRFs are represented by grey circles, of which there are 20 at each frequency point. Surrounding each cluster of measurements is the $95 \%$ confidence ellipse (assuming a bivariate normal distribution) [17].

The size and orientation of these ellipses provide an easy assessment of the associated FRF uncertainty. Under the (common) assumption of uncorrelated and identically distributed uncertainty, at a given frequency the distribution of measured FRFs and their associated confidence ellipse would form a circle around the mean value. From inspection of figures $1 \mathrm{~b}$ and $1 \mathrm{c}$ this is clearly not the case.

For both the point and transfer FRFs the confidence ellipse can be see to rotate with increasing frequency. This indicates that there not only exists a correlation between the real and imaginary components, but that this is dependent on frequency. It can further be noted that at resonance the maximum variance occurs in the imaginary component, which is in agreement with analysis presented above. 


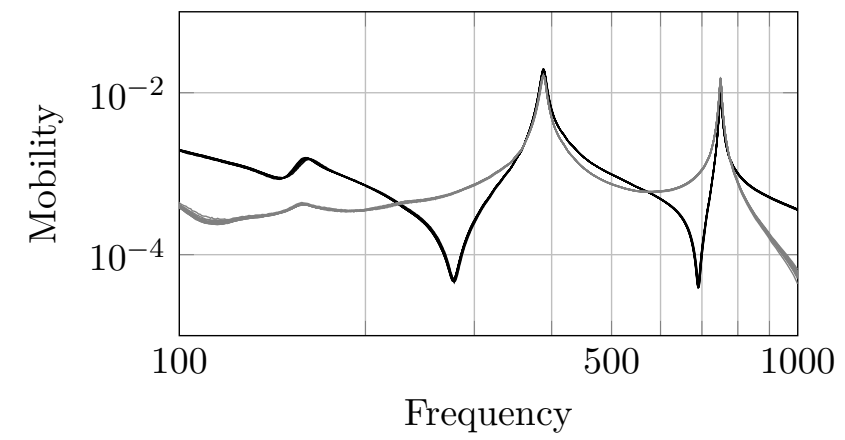

(a) Magnitude of the point and transfer FRF for 20 repeated excitations.

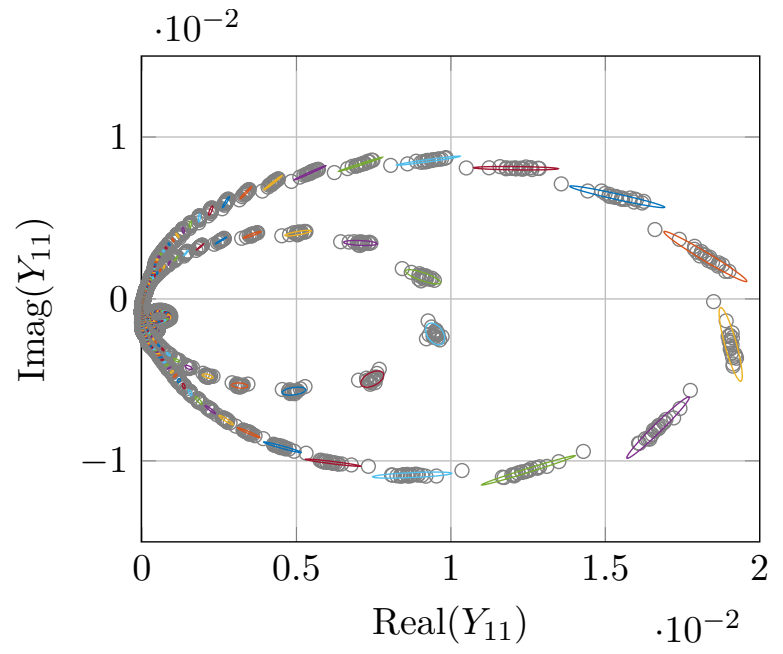

(b) Nyquist plot of the point FRF showing real and imaginary componenents of each excitation with their associated $95 \%$ confidence intervals.

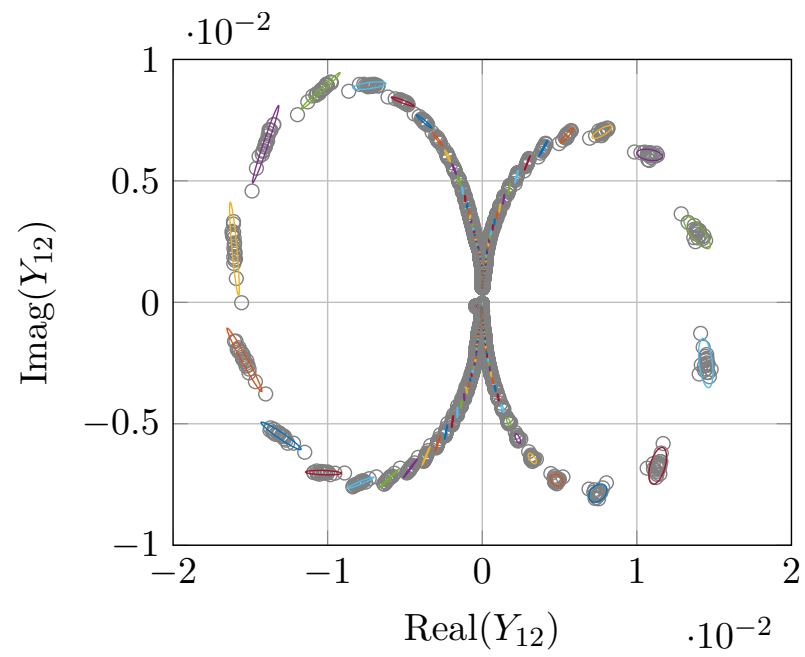

(c) Nyquist plot of the transfer FRF showing real and imaginary componenents of each excitation with their associated $95 \%$ confidence intervals.

Figure 1: Magnitude and bivariate uncertainty of point and transfer FRFs measured on a free-free beam using 20 repeated excitations.

To demonstrate that there further exists a correlation between the real and imaginary components of two simultaneously measured FRFs, the covariances: $\operatorname{Cov}\left[\mathfrak{R}\left(Y_{11}\right), \mathfrak{R}\left(Y_{21}\right)\right]$, $\operatorname{Cov}\left[\mathfrak{J}\left(Y_{11}\right), \mathfrak{J}\left(Y_{21}\right)\right]$, and $\operatorname{Cov}\left[\mathfrak{R}\left(Y_{11}\right), \mathfrak{J}\left(Y_{21}\right)\right]$ are plotted in fig- ure 2. Also shown in figure 3 are the corresponding correlation coefficients, defined as so,

$$
\operatorname{Corr}[A, B]=\frac{\operatorname{Cov}[A, B]}{\sigma_{A} \sigma_{B}} .
$$

Although the covariances a largely near zero, there are clear deviations about the resonant frequencies, i.e. around 160, 390 and $750 \mathrm{~Hz}$. These deviations are more clearly seen in the correlation coefficients of figure 3 .

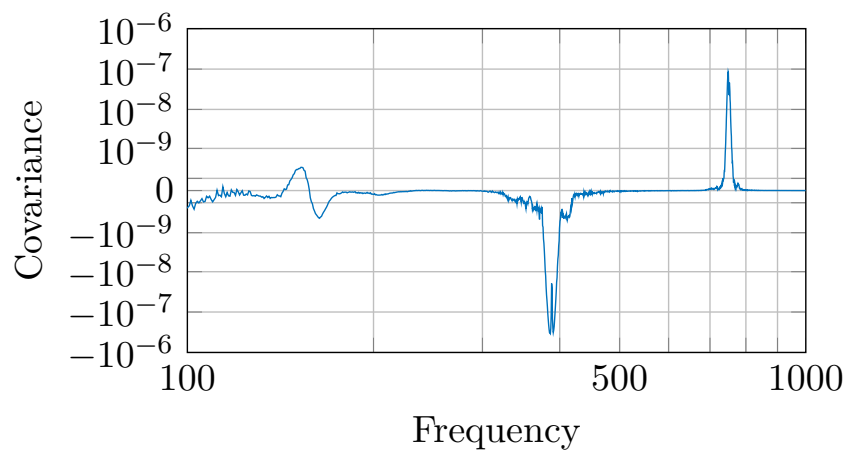

(a) Covariance between $\mathfrak{R}\left(Y_{11}\right)$ and $\mathfrak{R}\left(Y_{21}\right)$

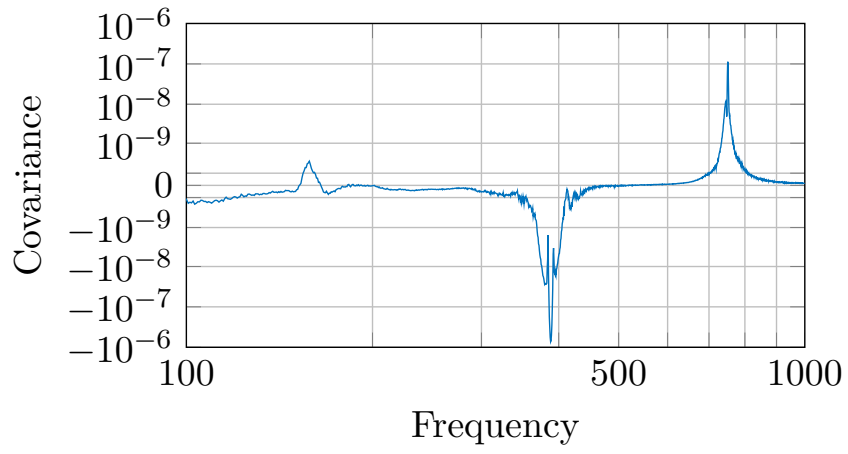

(b) Covariance between $\mathfrak{J}\left(Y_{11}\right)$ and $\mathfrak{J}\left(Y_{21}\right)$

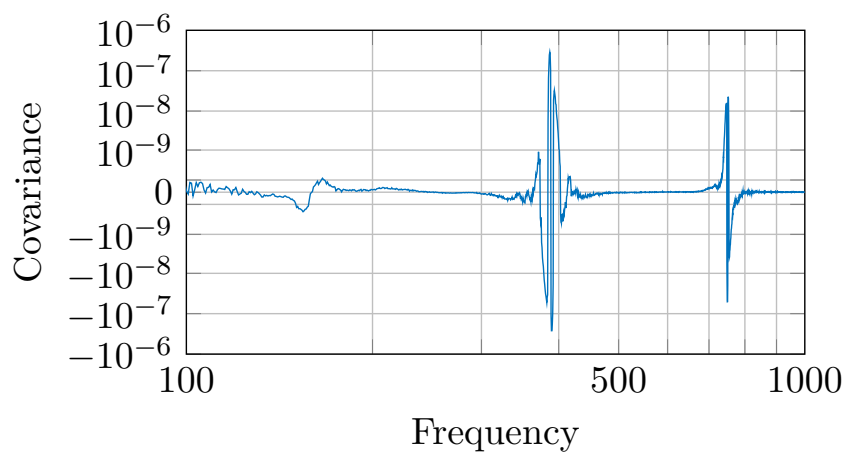

(c) Covariance between $\mathfrak{R}\left(Y_{11}\right)$ and $\mathfrak{J}\left(Y_{21}\right)$

Figure 2: Covariances between the real and imaginary components of a point and transfer FRF.

These correlations provide further evidence of operator uncertainty and raise the question as to whether the assumption of uncorrelated uncertainty that is typically assumed is a valid one. 
Whilst the above results demonstrate that there exist correlations between measured FRFs, their influence on the propagation of uncertainty has not yet been considered.

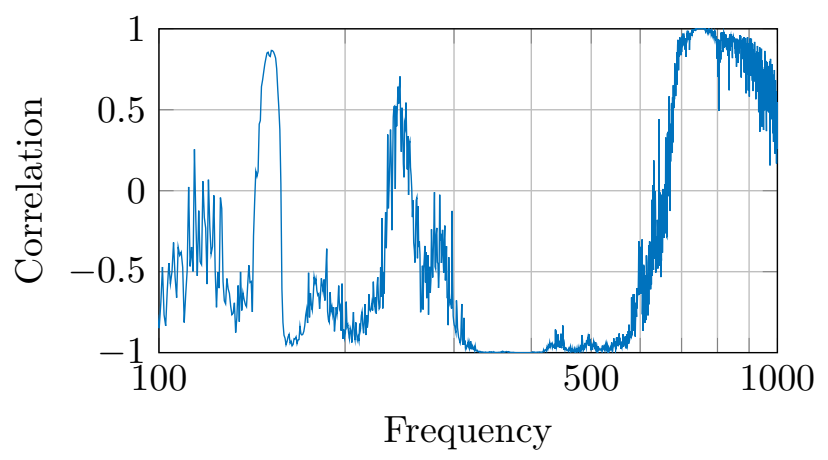

(a) Correlation coefficient between $\mathfrak{R}\left(Y_{11}\right)$ and $\mathfrak{R}\left(Y_{21}\right)$

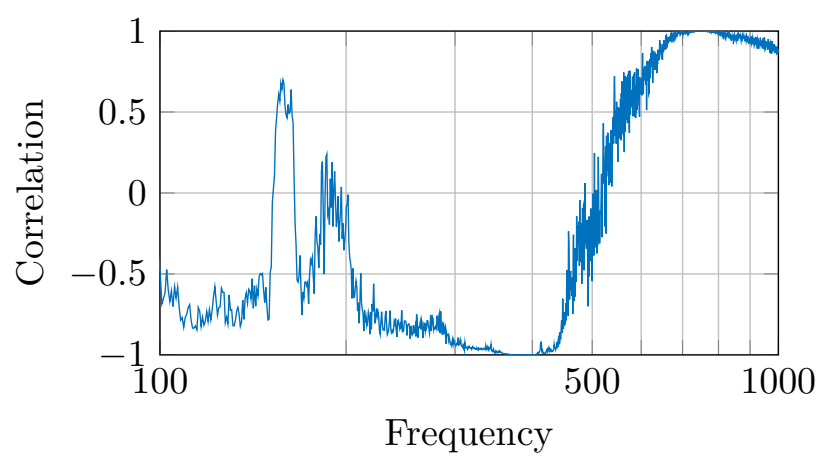

(b) Correlation coefficient between $\mathfrak{J}\left(Y_{11}\right)$ and $\mathfrak{I}\left(Y_{21}\right)$

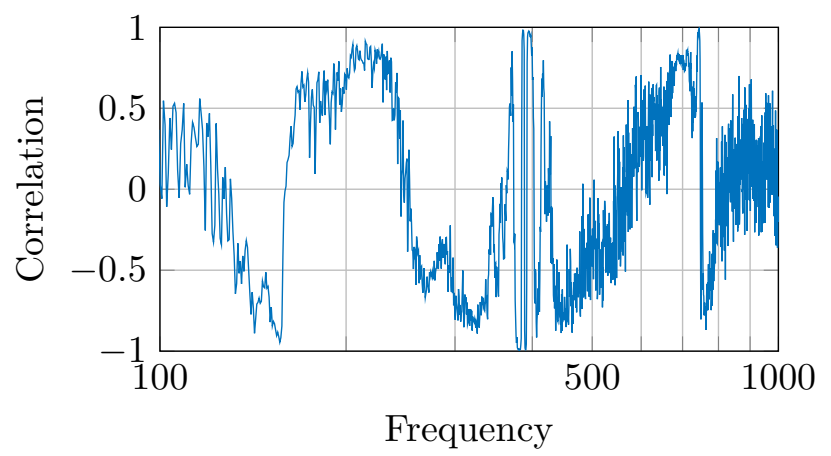

(c) Correlation coefficient between $\mathfrak{R}\left(Y_{11}\right)$ and $\mathfrak{J}\left(Y_{21}\right)$

Figure 3: Correlation coefficients between the real and imaginary components of a point and transfer FRF.

In the following section the influence of inter-FRF correlation on the propagation of uncertainty through a matrix inversion will be investigated. The inversion of a measured FRF matrix is a common procedure used often in experimental structural dynamics, and so is considered a representative example of FRF propagation.

\section{Numerical Study}

The aim of this study is to investigate the potential influence of inter-FRF correlation on some parameter estimation. Whilst there clearly exists a number of parameters that could be estimated from a set of measured FRFs, here we will limit our interest to those associated with matrix inversions. The inversion of a measured FRF matrix is an essential step in both DSS and inverse force identification procedures. With the aim of answering the question 'Do we need to account for inter-FRF correlation?', it is sufficient to investigate whether the propagation of uncertainty through a matrix inversion is sensitive the inclusion of inter-FRF correlation.

A diagrammatic representation of the numerical study is presented in figure 4. A numerical free-free beam model (see Appendix D of [22]) is excited externally at two positions, labelled 1 and 2 , each by a force-moment pair. The resulting (mobility) FRF matrix $\mathbf{Y} \in \mathbb{C}^{4 \times 4}$ is inverted to obtained the impedance matrix $\mathbf{Y}^{\mathbf{- 1}}=\mathbf{Z} \in \mathbb{C}^{4 \times 4}$. Operator uncertainty was modelled using randomly distributed excitation positions, centred about positions 1 and 2. The position of each force and moment excitation was generated according to a Gaussian distribution. A total of 10 excitations were applied at each degree of freedom (DoF). In keeping with experimental practice, response positions were kept fixed. The degree of operator uncertainty was set by the variance of the excitation distribution, $\sigma^{2}$. This corresponds roughly to a $95 \%$ probability of exciting within $\pm \sigma \times 1.96$ of the intended position. For clarity, the effect of measurement uncertainty has not been included here.

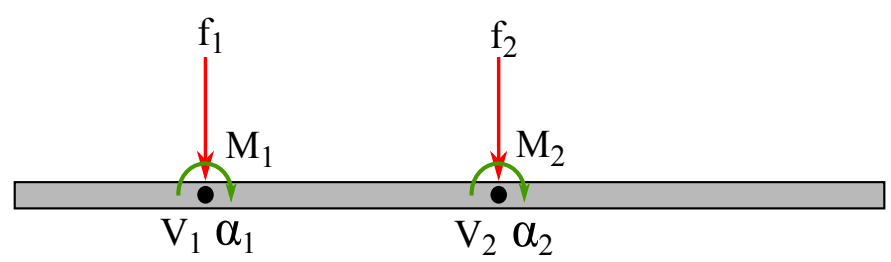

Figure 4: Diagrammatic representation of numerical simulation.

We are concerned with the uncertainty present in an (arbitrary) element of the impedance matrix when the inter-FRF correlations have, and have not, been included in the propagation of uncertainty.

The propagation of uncertainty on measured FRFs through a matrix inversion was considered by Meggitt et al. [22], where a linearised relation was derived, relating the covariance of measured FRFs, to those of its inverse. An alternative derivation of this relation, for the special case of complex square matrices is given in Appendix B. Referred to as a Linear Inverse Propagation (LIP), the relations of note are given by,

$$
\begin{gathered}
\operatorname{Cov}\left[\mathfrak{R}\left(Z_{i j}\right), \mathfrak{R}\left(Z_{l m}\right)\right]=\frac{1}{4} \sum_{a, b, c, d}\left(Z_{i a} Z_{b j} Z_{l c} Z_{d m} \operatorname{Cov}\left[Y_{a b}, Y_{c d}\right]+\right. \\
Z_{i a} Z_{b j} Z_{l c}^{*} Z_{d m}^{*} \operatorname{Cov}\left[Y_{a b}, Y_{c d}^{*}\right]+Z_{i a}^{*} Z_{b j}^{*} Z_{l c} Z_{d m} \operatorname{Cov}\left[Y_{a b}^{*}, Y_{c d}\right]+ \\
\left.Z_{i a}^{*} Z_{b j}^{*} Z_{l c}^{*} Z_{d m}^{*} \operatorname{Cov}\left[Y_{a b}^{*}, Y_{c d}^{*}\right]\right),
\end{gathered}
$$




$$
\begin{gathered}
\operatorname{Cov}\left[\mathfrak{J}\left(Z_{i j}\right), \mathfrak{J}\left(Z_{l m}\right)\right]=-\frac{1}{4} \sum_{a, b, c, d}\left(Z_{i a} Z_{b j} Z_{l c} Z_{d m} \operatorname{Cov}\left[Y_{a b}, Y_{c d}\right]-\right. \\
Z_{i a} Z_{b j} Z_{l c}^{*} Z_{d m}^{*} \operatorname{Cov}\left[Y_{a b}, Y_{c d}^{*}\right]-Z_{i a}^{*} Z_{b j}^{*} Z_{l c} Z_{d m} \operatorname{Cov}\left[Y_{a b}^{*}, Y_{c d}\right]+ \\
\left.Z_{i a}^{*} Z_{b j}^{*} Z_{l c}^{*} Z_{d m}^{*} \operatorname{Cov}\left[Y_{a b}^{*}, Y_{c d}^{*}\right]\right),
\end{gathered}
$$

$$
\begin{gathered}
\operatorname{Cov}\left[\Re\left(Z_{i j}\right), \mathfrak{J}\left(Z_{l m}\right)\right]=\frac{1}{i 4} \sum_{a, b, c, d}\left(Z_{i a} Z_{b j} Z_{l c} Z_{d m} \operatorname{Cov}\left[Y_{a b}, Y_{c d}\right]-\right. \\
Z_{i a} Z_{b j} Z_{l c}^{*} Z_{d m}^{*} \operatorname{Cov}\left[Y_{a b}, Y_{c d}^{*}\right]+Z_{i a}^{*} Z_{b j}^{*} Z_{l c} Z_{d m} \operatorname{Cov}\left[Y_{a b}^{*}, Y_{c d}\right]- \\
\left.Z_{i a}^{*} Z_{b j}^{*} Z_{l c}^{*} Z_{d m}^{*} \operatorname{Cov}\left[Y_{a b}^{*}, Y_{c d}^{*}\right]\right),
\end{gathered}
$$

and

$$
\begin{gathered}
\operatorname{Cov}\left[\mathfrak{I}\left(Z_{i j}\right), \mathfrak{R}\left(Z_{l m}\right)\right]=\frac{1}{i 4} \sum_{a, b, c, d}\left(Z_{i a} Z_{b j} Z_{l c} Z_{d m} \operatorname{Cov}\left[Y_{a b}, Y_{c d}\right]-\right. \\
Z_{i a}^{*} Z_{b j}^{*} Z_{l c} Z_{d m} \operatorname{Cov}\left[Y_{a b}^{*}, Y_{c d}\right]+Z_{i a} Z_{b j} Z_{l c}^{*} Z_{d m}^{*} \operatorname{Cov}\left[Y_{a b}, Y_{c d}^{*}\right]- \\
\left.Z_{i a}^{*} Z_{b j}^{*} Z_{l c}^{*} Z_{d m}^{*} \operatorname{Cov}\left[Y_{a b}^{*}, Y_{c d}^{*}\right]\right),
\end{gathered}
$$

where, $Z_{i j}$ is the $i$ th element of the expected impedance matrix (acquired through the inverse of the expected mobility matrix), $Y_{i j}$ is the $i j$ th element of the mobility matrix (which may be considered a matrix of RVs), and * represents complex conjugation. Note that the equations 23-26 are based on a complex covariance (i.e. $Y_{a b}$ and $Y_{c d}$ are in their complex form) and do not require explicitly a bivariate covariance matrix. An alternative, more general, formulation is presented in [22] based on the mobility's bivariate covariance matrix.

The above state that the complex bivariate uncertainty in an element of the impedance matrix is given by the quadruple sum over all elements of the mobility variance-covariance matrix, each weighted by a product of expected impedances and conjugated appropriately. These relations were derived by considering the first order expansion of a perturbed matrix inversion. As such, they are appropriate for small uncertainty only. Nevertheless, their form allows us to easily neglect the influence of inter-FRF correlation by assuming $\operatorname{Cov}\left[Y_{a b}, Y_{c d}\right]=0$, for all $a b \neq c d$.

A Monte-Carlo (MC) simulation is used to provide a correct propagation of uncertainty for comparison. The MC simulation involves the repeated inversion of realisations of the mobility matrix, each built from an independent combination of the measured mobilities. The resulting impedances matrices are subsequently used to estimate the variance-covariance matrix, $\boldsymbol{\Sigma}_{\mathbf{Z}}$. The MC simulation implicitly includes the effect of any correlations that exist between the mobilities, whilst avoiding the need to linearise the problem, thus capturing the non-linear mapping present in the matrix inversion.

Shown in figure 5 are the predicted (bivariate) uncertainties of the impedance element $Z_{11}$, for an operator uncertainty of $\sigma=10^{-5}$, in the form of their relative variance and covariance, defined, respectively, as,

$$
\operatorname{RelVar}[A]=\frac{\operatorname{Var}[A]}{\mathbb{E}[A]^{2}}
$$

and

$$
\operatorname{Rel} \operatorname{Cov}[A, B]=\frac{\operatorname{Cov}[A, B]}{\mathbb{E}[A] \mathbb{E}[B]} .
$$

In blue are the uncertainties acquired through a $\mathrm{MC}$ simulation, which involved the inversion of 1000 mobility matrix realisations. In orange are the uncertainties predicted through the LIP approach (including inter-FRF correlation). In green are the uncertainties acquired through the LIP approach when inter-FRF correlation is neglected.

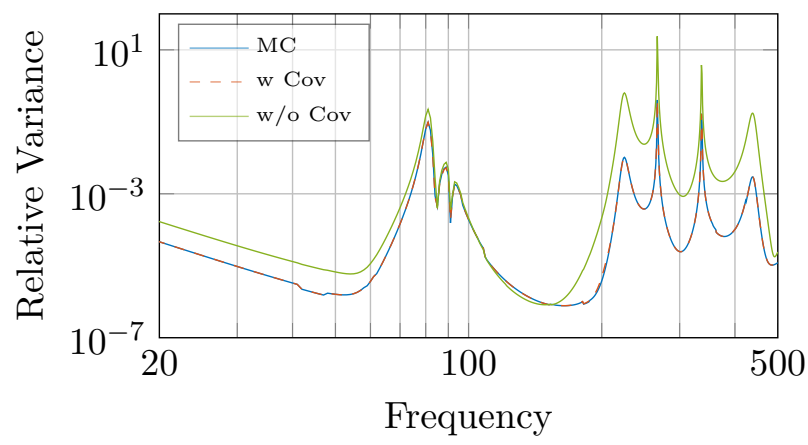

(a) Relative variance of $\mathfrak{R}\left(Z_{11}\right)$

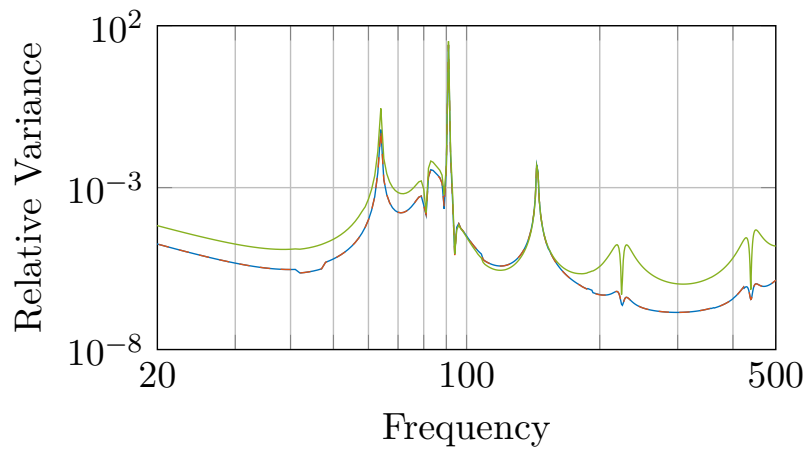

(b) Relative variance of $\mathfrak{J}\left(Z_{11}\right)$

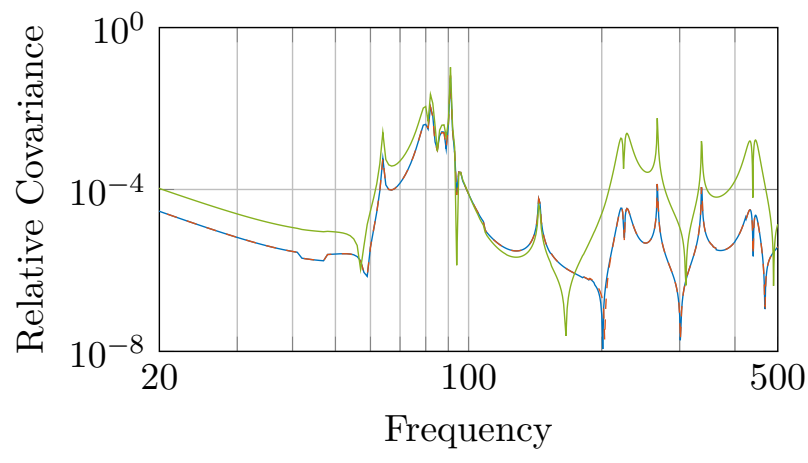

(c) Relative covariance between $\mathfrak{R}\left(Z_{11}\right)$ and $\mathfrak{J}\left(Z_{11}\right)$

Figure 5: Complex bivariate uncertainty of the impedance element $Z_{11}$ due to operator uncertainty. Calculated using Monte-Carlo propagation (in blue) and the Linear Inverse Propagation method, with (in orange) and without (in green) mobility covariances. The excitation distribution was $\sigma=10^{-5}$.

The results clearly illustrate that the neglect of correlation between the measured mobilities can have a severe effect on the 
estimated impedance uncertainty. Inclusion of the inter-FRF correlation can be seen to yield an uncertainty estimate in excellent agreement with that of the MC simulation, deviating only marginally in some regions (this would be expected given the linear assumption the LIP approach is based on). These results further demonstrate the LIPs ability to propagated complex and correlated uncertainty through a matrix inversion.

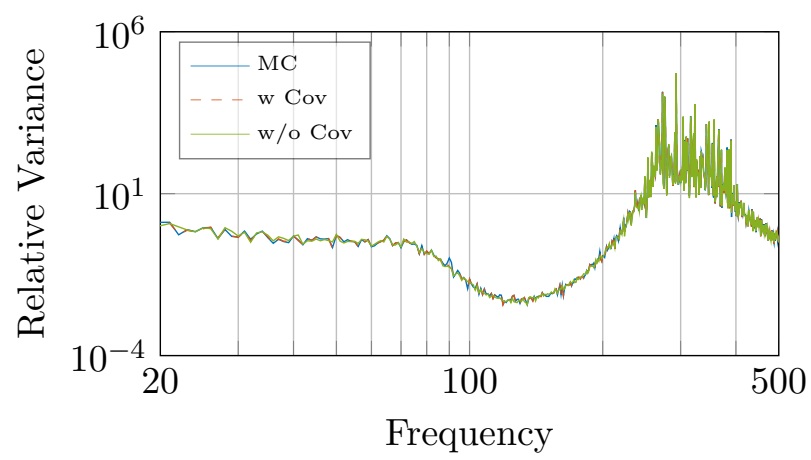

(a) Relative variance of $\mathfrak{R}\left(Z_{11}\right)$

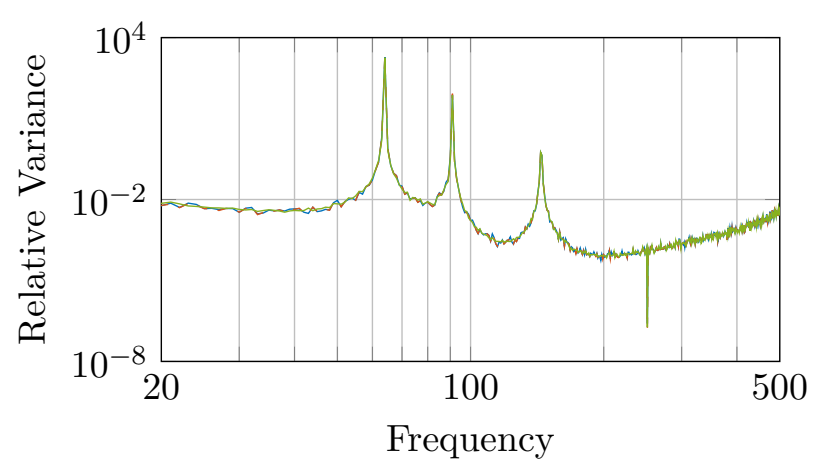

(b) Relative variance of $\mathfrak{J}\left(Z_{11}\right)$

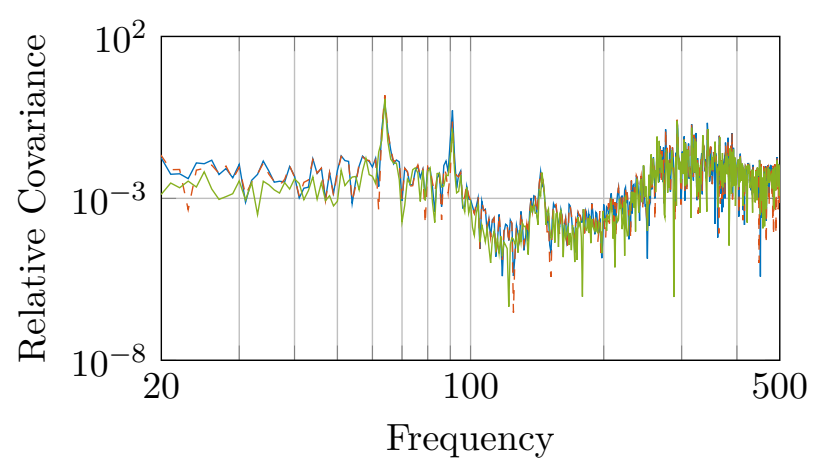

(c) Relative covariance between $\mathfrak{R}\left(Z_{11}\right)$ and $\mathfrak{J}\left(Z_{11}\right)$

Figure 6: Complex bivariate uncertainty of the impedance element $Z_{11}$ due to measurement uncertainty. The signal to noise ratio (SNR) of each mobility element was $30 \mathrm{~dB}$. Legend as in figure 5 .

It is worth noting that the LIP predictions 'with covariance' also account for the covariance between mobilities of different excitation types/positions. The level of this covariance was typically on the order of $10^{-28}$ and an identical set of results were obtained when this covariance was neglected. This is in agree- ment with the notion that the underlying operator uncertainty is uncorrelated between excitation positions $(\operatorname{Cov}[\mathbf{a}, \mathbf{d}]=0)$.

Shown in figure 6 are the impedance uncertainty estimates in the case of an additive Gaussian noise on the mobility (i.e. measurement uncertainty), as opposed to operator uncertainty. In this case the elements of the mobility matrix are uncorrelated with one another. As a result, the neglect of inter-FRF correlation has a minimal effect on the propagation of uncertainty. This result supports the use of the simplified DSS propagation framework in [8] in the case of measurement uncertainty only. Figure 5 however, demonstrates that in the presence of operator uncertainty, inter-FRF correlation must be accounted for.

Further to the propagation of uncertainty in DSS, the above results are of relevance to inverse problems of the form $\mathbf{A x}=\mathbf{b}$, where $\mathbf{b}$ represents an observable output of a system governed by the matrix $\mathbf{A}$, due to some unknown input $\mathbf{x}$. Such problems are routinely encountered in fields as diverse as physics, geophysics, engineering and finance. The present results suggest that inter-element correlation within the matrix $\mathbf{A}$ can contribute significantly to the uncertainty in the resultant solution, and must therefore be accounted for if present.

Although a simple numerical study, similar effects have been observed in experimental work by the author, see for example section 4 .

\section{Experimental Study}

To further illustrate both the presence and influence of interFRF correlation, a brief experimental study is presented. In this study, a 3 footed vibration source is rigidly coupled to a large steel plate, as illustrated in figure 7. The aim of the study is to measure the source-receiver interface FRF matrix, determine its inverse, and investigate the influence of inter-FRF correlation on its uncertainty (similar to the numerical study presented in section 3). The measurement of such an interface FRF matrix is an essential step in many analyses, for example, vibration source characterisation, dynamic sub-structuring, transfer path analysis [27], and experimental modal analysis (to name but a few), and is therefore of some practical relevance.

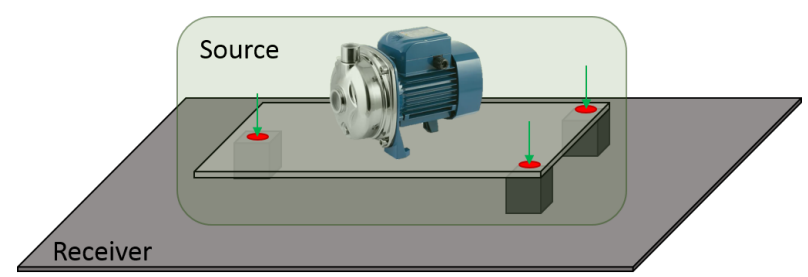

Figure 7: Diagrammatic representation of experimental study. Green arrows and red circles correspond to excitation and response positions, respectively.

For simplicity we will consider only the vertical $\mathrm{Z}$ interface DoFs. Consequently, the measured FRF (mobility) matrix, $\mathbf{Y} \in \mathbb{C}^{3 \times 3}$, is $3 \times 3$. Each measurement position was excited 20 times, from which an appropriate covariance matrix was estimated. This covariance matrix was then propagated through a 
matrix inversion as per equations 23-26. This was done both with and without inter-FRF correlation, the latter achieved by setting all but the diagonal covariance elements to 0 . To provide a correct propagation of uncertainty (including non-linear and inter-FRF effects) a Monte-Carlo simulation was performed. Note that due to the high modal density of the structure considered, results are presented over a frequency range of 100 to $500 \mathrm{~Hz}$. Outside of this range similar results are obtained.

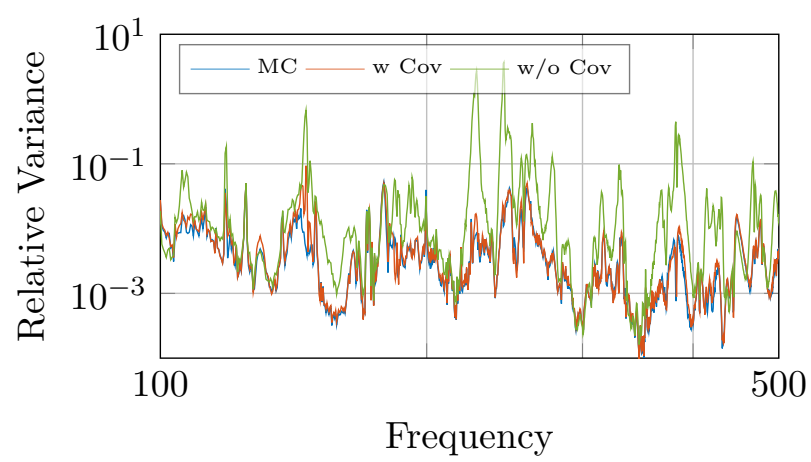

(a) Relative variance of $\mathfrak{R}\left(Z_{11}\right)$

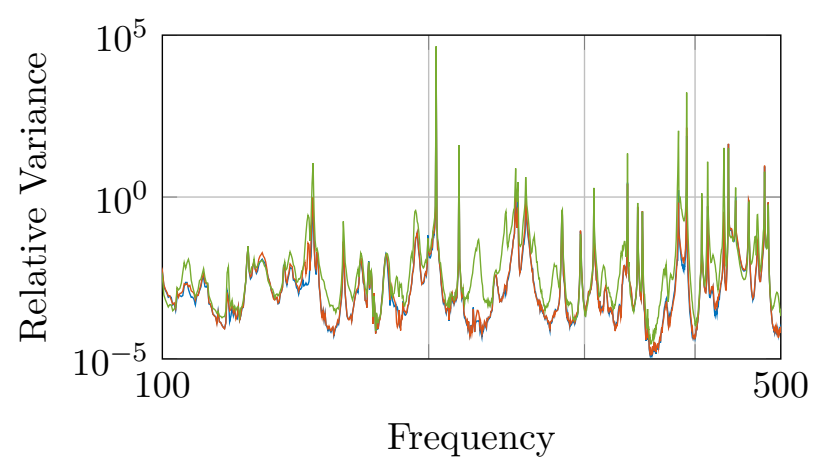

(b) Relative variance of $\mathfrak{J}\left(Z_{11}\right)$

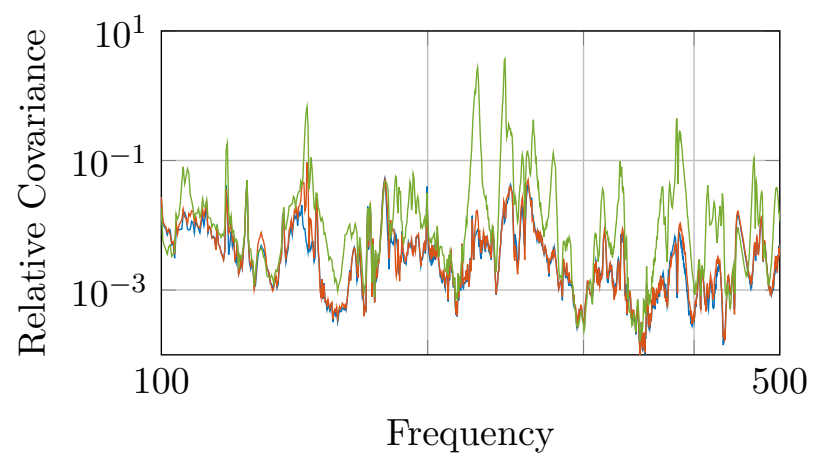

(c) Relative covariance between $\mathfrak{R}\left(Z_{11}\right)$ and $\mathfrak{J}\left(Z_{11}\right)$

Figure 8: Complex bivariate uncertainty of the impedance element $Z_{11}$ from the experimental study. Calculated using Monte-Carlo propagation (in blue) and the Linear Inverse Propagation method, with (in orange) and without (in green) mobility covariances.

Shown in figure 8 are the predicted (bivariate) uncertainties of the impedance element $Z_{11}$ in the form of their relative variance and covariance. As in the numerical study presented in section 3, the neglect of inter-FRF correlation is seen to lead to an over estimation of uncertainty in particular regions of the response. When included, the inter-FRF correlations can be seen to yield an uncertainty estimate in excellent agreement with the MC simulation.

The results of figure 8 are reproduced in figure 9, where the magnitude of the impedance element $Z_{11}$ is shown alongside a pair of $95 \%$ confidence intervals. These confidence intervals were obtained by propagating the bivariate uncertainty of $Z_{11}$ onto its magnitude, as per equation 28 of [22]. The magnitude variance was then used to compute the confidence interval, for an assumed log-normal distribution, based on Appendix $\mathrm{C}$ of [28]. From figure 9 it is clear that a neglect of inter-FRF correlation can lead to a reduced confidence in the resultant impedance. This result clearly illustrates the importance of inter-FRF correlation when propagating uncertainty.

Based on the result presented above it is argued that a more general treatment of FRF uncertainty is required if uncertainties are to be handled and propagated correctly, for example in the estimation of some parameter. In particular, the inter-FRF correlation arising due to operator uncertainty has been shown to influence greatly the propagation of uncertainty, and must therefore be accounted for.

\section{Conclusion}

Frequency response functions (FRFs) are routinely used within experimental structural dynamics, and have become an essential requirement in numerous analyses and standardised procedures. As of yet, however, few works have considered generally the treatment of their uncertainty. It is often assumed that measured FRFs are polluted by uncorrelated noise, and are therefore uncorrelated themselves. In the present paper it has been shown that the notion of operator uncertainty (i.e. inconsistency in excitation position and/or orientation) introduces a correlation between simultaneously measured FRFs. The importance of this inter-FRF correlation on the propagation of uncertainty (for example in the estimation of some parameter) was demonstrated by considering the inversion of a 'measured' FRF matrix as part of a numerical and experimental study. It was shown that in the presence of inter-FRF correlation, large errors can arise in the propagated uncertainty estimates if such correlations are neglected. To this end a set of Linearised Inverse Propagation relations were introduced which facilitate the propagation of correlated uncertainty through a matrix inversion. Whilst this result applies directly only to procedures that involve a matrix inversion, similar errors will likely emerge through other procedures, for example, modal parameter estimation.

It is therefore argued that a general treatment of FRF uncertainty warrants a complex bivariate description that accounts for all inter-FRF correlations that exists between simultaneously measured FRFs.

\section{Appendix A. Estimation of an FRF Covariance Matrix}

The estimation of $\boldsymbol{\Sigma}_{\mathbf{H}}$ may be performed in a number of ways. In any case, each position of interest must be excited re- 


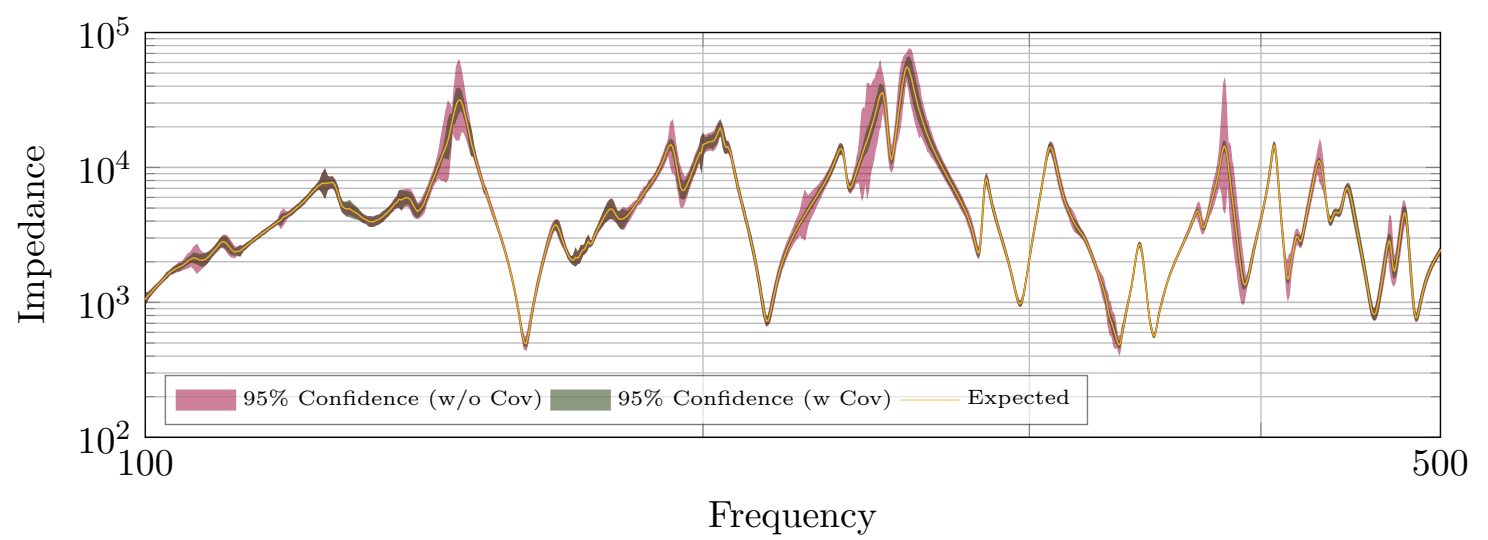

Figure 9: Magnitude of impedance element $Z_{11}$ with 95\% confidence bounds, for an assumed log-normal distribution. The red and green confidence intervals are obtained by neglecting and including inter-FRF correlation in the propagation of uncertainty, respectively.

peatedly such that a series of $P$ measurements are made. The user must then decide at what point in the preceding analysis to estimate the statistical properties of the measurements made. As an example, one may consider the complex Fourier spectra of the input and output signals as the initial RVs and determine their associated variance covariance matrices. To then acquire the variance covariance matrix of an FRF the Fourier spectra uncertainty must be propagated through the H1 (or H2) estimator function. Alternatively, one may consider the FRF itself as the RV and determine the FRF variance covariance matrix directly, thus avoiding the need to perform additional uncertainty propagations. This may be beneficial as each stage of propagation incurs an error since linearity of the propagation function is assumed.

Let us consider the latter approach. Suppose we measure the FRF matrix $\mathbf{H} \in \mathbb{C}^{M \times N}$, repeating each excitation $P$ times. The resulting FRFs may be arranged as so,

$$
\hat{\mathbf{H}}=\left[\begin{array}{ccc|c|ccc}
\mathbf{H}_{11}^{(\mathbf{1})} & \cdots & \mathbf{H}_{\mathbf{M 1}}^{(\mathbf{1} 1} & \cdots & \mathbf{H}_{\mathbf{1 N}}^{(\mathbf{1})} & \cdots & \mathbf{H}_{\mathbf{N N}}^{(\mathbf{1})} \\
\mathbf{H}_{11}^{(\mathbf{2})} & \cdots & \mathbf{H}_{\mathbf{M 1}}^{(\mathbf{2} 1} & \cdots & \mathbf{H}_{\mathbf{1 N}}^{(\mathbf{2})} & \cdots & \mathbf{H}_{\mathbf{N N}}^{(\mathbf{2})} \\
\vdots & \vdots & \vdots & \vdots & \vdots & \vdots & \vdots \\
\mathbf{H}_{\mathbf{1 1}}^{(\mathbf{P})} & \cdots & \mathbf{H}_{\mathbf{M 1}}^{(\mathbf{P})} & \cdots & \mathbf{H}_{\mathbf{1 N}}^{(\mathbf{P})} & \cdots & \mathbf{H}_{\mathbf{M N}}^{(\mathbf{P})}
\end{array}\right]
$$

where $\mathbf{H}_{\mathrm{ij}}^{(\mathbf{P})}=\left[\mathfrak{R}\left(H_{i j}^{(P)}\right) \quad \mathfrak{J}\left(H_{i j}^{(P)}\right)\right]$ is the $P$ th measurement of the FRF describing the response at $i$ due to an excitation at $j$. The matrix $\hat{\mathbf{H}} \in \mathbb{C}^{P \times 2 N M}$ could then used to estimate the covariance matrix $\boldsymbol{\Sigma}_{\mathbf{H}}$. In the context of a covariance estimation, each row of $\hat{\mathbf{H}}$ may be thought of as an 'observation' of the FRF matrix $\mathbf{H}$. Note that,

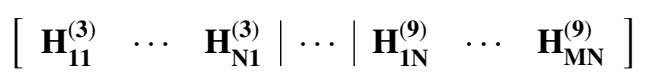

say, is an equally valid observation, since the excitations at positions 1 and $N$ are independent events. One may be tempted to supplement $\hat{\mathbf{H}}$ with additional (perhaps all possible) observations by considering different combinations of excitations. Adding addition observations in this way would only aid in estimating the correlation between different excitation positions, since no additional information is introduced regarding the FRFs of shared excitation position. However, noting that the excitations at each pair of positions (say, 1 and $N$ ) are uncorrelated, adding addition observations will simply aid in the (interpositional) covariance terms tending to zero. Alternatively, the variance covariance matrix $\boldsymbol{\Sigma}_{\mathbf{H}}$ may be constructed by considering the variance covariance matrix of each column of $\mathbf{H}$ in turn, and block diagonalising them as so,

$$
\Sigma_{H}=\left[\begin{array}{ccc}
\Sigma_{H_{: 1}} & 0 & 0 \\
0 & \ddots & 0 \\
0 & 0 & \Sigma_{H_{: N}}
\end{array}\right]
$$

where $\boldsymbol{\Sigma}_{\mathbf{H}_{: \mathbf{N}}}$ is the bivariate variance covariance matrix of the $N$ th column of $\mathbf{H}$, estimated over the $P$ repeated measurements.

In its standard form $\boldsymbol{\Sigma}_{\mathbf{H}}$ describes the statistical dependencies between the set of repeated measurements. Whilst this may be of interest, it is typical to consider instead the uncertainty in the mean value of the repeated measurements. In this case a $1 / P$ normalisation should be applied to $\boldsymbol{\Sigma}_{\mathbf{H}}$. This normalised uncertainty may then be propagated as normal.

\section{Appendix B. Linear Inverse Propagation}

In what follows we will derive the complex bivariate forms of a Linear Inverse Propagation (LIP) relation. We begin by considering the measured mobility matrix, $\mathbf{Y}_{\mathbf{n}} \in \mathbb{C}^{N \times N}$, as the perturbation of a true mobility matrix $\mathbf{Y}$ in the form,

$$
\mathbf{Y}_{\mathbf{n}}=\mathbf{Y}+\Delta_{\mathbf{Y}_{\mathbf{n}}}
$$

where $\Delta_{\mathbf{Y}_{\mathbf{n}}} \in \mathbb{C}^{N \times N}$ is some small random deviation resulting from experimental error, and subscript $n$ denotes the $n$th mobility matrix out of a series of repeated measurements.

For a small $\Delta_{\mathbf{Y}_{\mathbf{n}}}$ the matrix inverse, $\mathbf{Y}_{\mathbf{n}}^{-\mathbf{1}}$, may be approximated to first order by,

$$
\left[\mathbf{Y}+\Delta_{\mathbf{Y}_{\mathbf{n}}}\right]^{-1} \approx \mathbf{Y}^{-\mathbf{1}}-\mathbf{Y}^{-\mathbf{1}} \Delta_{\mathbf{Y}_{\mathbf{n}}} \mathbf{Y}^{-\mathbf{1}}
$$

In context, equation B. 2 states that for a sufficiently small perturbation, the measured mobility matrix $\mathbf{Y}_{\mathbf{n}}$ yields an impedance 
of the form $\mathbf{Z}_{\mathbf{n}}=\mathbf{Z}-\mathbf{Z} \boldsymbol{\Delta}_{\mathbf{Y}_{\mathbf{n}}} \mathbf{Z}$, where $\mathbf{Z}$ is the true impedance matrix, and $-\mathbf{Z} \boldsymbol{\Delta}_{\mathbf{Y}_{\mathbf{n}}} \mathbf{Z}$ is the resultant perturbation,

$$
\Delta_{\mathbf{Z}_{\mathbf{n}}}=\mathbf{Z}_{\mathbf{n}}-\mathbf{Z}=-\mathbf{Z} \Delta_{\mathbf{Y}_{\mathbf{n}}} \mathbf{Z} .
$$

Rewriting equation B.3 as an element by element summation whilst taking its real part yields,

$$
\mathfrak{R}\left(\Delta_{Z_{n, i j}}\right)=-\sum_{a, b} \mathfrak{R}\left(Z_{i a} \Delta_{Y_{n, a b}} Z_{b j}\right)
$$

Multiplying both sides of equation B.4 by $\frac{1}{N-1} \mathfrak{J}\left(\Delta_{Z_{n, l m}}\right)$ whilst summing over $N$ measurements yields,

$$
\begin{aligned}
& \frac{1}{N-1} \sum_{n}^{N} \mathfrak{R}\left(\Delta_{Z_{n, i j}}\right) \mathfrak{J}\left(\Delta_{Z_{n, l m}}\right)= \\
& \frac{1}{N-1} \sum_{n}^{N} \sum_{a, b, c, d} \mathfrak{R}\left(Z_{i a} \Delta_{Y_{n, a b}} Z_{b j}\right) \mathfrak{J}\left(Z_{l c} \Delta_{Y_{n, c d}} Z_{d m}\right) .
\end{aligned}
$$

Noting that $\mathfrak{R}(x)=\frac{1}{2}\left(x+x^{*}\right)$ and that $\mathfrak{I}(x)=\frac{1}{i 2}\left(x-x^{*}\right)$, where $*$ represents complex conjugation, the RHS of equation B.5 may be separated as,

$$
\begin{gathered}
\frac{1}{N-1} \sum_{n}^{N} \mathfrak{R}\left(\Delta_{Z_{n, i j}}\right) \mathfrak{J}\left(\Delta_{Z_{n, l m}}\right)=\frac{1}{N-1} \frac{1}{i 4} \sum_{n}^{N} \sum_{a, b, c, d} \\
\left(Z_{i a} \Delta_{Y_{n, a b}} Z_{b j}+Z_{i a}^{*} \Delta_{Y_{n, a b}}^{*} Z_{b j}^{*}\right)\left(Z_{l c} \Delta_{Y_{n, c d}} Z_{d m}-Z_{l c}^{*} \Delta_{Y_{n, c d}}^{*} Z_{d m}^{*}\right) .
\end{gathered}
$$

Expanding the bracketed terms we arrive at,

$$
\begin{gathered}
\frac{1}{N-1} \sum_{n}^{N} \mathfrak{R}\left(\Delta_{Z_{n, i j}}\right) \mathfrak{J}\left(\Delta_{Z_{n, l m}}\right)=\frac{1}{N-1} \frac{1}{i 4} \sum_{n}^{N} \sum_{a, b, c, d} \\
\left(Z_{i a} Z_{b j} Z_{l c} Z_{d m} \Delta_{Y_{n, a b}} \Delta_{Y_{n, c d}}-Z_{i a} Z_{b j} Z_{l c}^{*} Z_{d m}^{*} \Delta_{Y_{n, a b}} \Delta_{Y_{n, c d}}^{*}+\right. \\
\left.Z_{i a}^{*} Z_{b j}^{*} Z_{l c} Z_{d m} \Delta_{Y_{n, a b}}^{*} \Delta_{Y_{n, c d}}-Z_{i a}^{*} Z_{b j}^{*} Z_{l c}^{*} Z_{d m}^{*} \Delta_{Y_{n, a b}}^{*} \Delta_{Y_{n, c d}}^{*}\right) .
\end{gathered}
$$

A statistical parallel may now be drawn by noting that the covariance between the real and imaginary components of any two elements of the impedance matrix is given by,

$$
\operatorname{Cov}\left[\mathfrak{R}\left(Z_{i j}\right), \mathfrak{J}\left(Z_{l m}\right)\right]=\frac{1}{N-1} \sum_{n}^{N} \mathfrak{R}\left(\Delta_{Z_{n, i j}}\right) \mathfrak{J}\left(\Delta_{Z_{n, l m}}\right)
$$

and that similarly, the covariance between any two elements of the mobility matrix is given by,

$$
\operatorname{Cov}\left[Y_{a b}, Y_{c d}\right]=\frac{1}{N-1} \sum_{n}^{N} \Delta_{Y_{n, a b}} \Delta_{Y_{n, c d}} .
$$

Substituting into equation B.7, the bivariate linearised propagation of uncertainty through a matrix inversion may be expressed by the relation,

$$
\begin{aligned}
& \operatorname{Cov}\left[\mathfrak{R}\left(Z_{i j}\right), \mathfrak{J}\left(Z_{l m}\right)\right]=\frac{1}{i 4} \sum_{a, b, c, d}\left(Z_{i a} Z_{b j} Z_{l c} Z_{d m} \operatorname{Cov}\left[Y_{a b}, Y_{c d}\right]-\right. \\
& Z_{i a} Z_{b j} Z_{l c}^{*} Z_{d m}^{*} \operatorname{Cov}\left[Y_{a b}, Y_{c d}^{*}\right]+Z_{i a}^{*} Z_{b j}^{*} Z_{l c} Z_{d m} \operatorname{Cov}\left[Y_{a b}^{*}, Y_{c d}\right]- \\
& \left.Z_{i a}^{*} Z_{b j}^{*} Z_{l c}^{*} Z_{d m}^{*} \operatorname{Cov}\left[Y_{a b}^{*}, Y_{c d}^{*}\right]\right) \text {. }
\end{aligned}
$$

Equation B.10 describes the covariance between the real and imaginary components of any two elements of the impedance matrix in terms of the true impedance, the mobility covariance, and their conjugates. The impedance terms in equation B.10 correspond to those of the true impedance matrix and must be replaced by the expected values obtained through the inversion of an expected mobility matrix. This requires the further assumption that the perturbation matrix has a zero mean, $\mathbb{E}\left[\boldsymbol{\Delta}_{\mathbf{Y}_{\mathbf{n}}}\right]=\mathbf{0}$.

Following a similar procedure to the above, the covariances, $\operatorname{Cov}\left[\mathfrak{R}\left(Z_{i j}\right), \mathfrak{R}\left(Z_{l m}\right)\right], \operatorname{Cov}\left[\mathfrak{J}\left(Z_{i j}\right), \mathfrak{J}\left(Z_{l m}\right)\right]$, and $\operatorname{Cov}\left[\mathfrak{J}\left(Z_{i j}\right), \mathfrak{R}\left(Z_{l m}\right)\right]$ may readily be found (see equations $23-26$ ).

Lastly, in the special case that $\mathbf{Y}_{\mathbf{n}} \in \mathbb{R}^{N \times N}$ is a real square matrix, note that the above derivation will reduce to,

$$
\operatorname{Cov}\left[Z_{i j}, Z_{l m}\right]=\sum_{a, b, c, d} Z_{i a} Z_{b j} Z_{l c} Z_{d m} \operatorname{Cov}\left[Y_{a b}, Y_{c d}\right]
$$

which is in exact agreement with that of [29]. Equations 2326 may therefore be considered a generalisation of [29] to the complex bivariate case.

\section{Acknowledgements}

This work was funded through the EPSRC Research Grant EP/P005489/1 Design by Science. The author would like to acknowledge A.T. Moorhouse and A.S. Elliott from The University of Salford, and A. Clot and R.S. Langley from The University of Cambridge for their involvement in this work.

\section{References}

[1] D.J Ewins. Modal Testing: Theory, practise and application. Research Studies Press LTD, 2 edition, 2000.

[2] P. Sjövall and T. Abrahamsson. Component system identification and state-space model synthesis. Mechanical Systems and Signal Processing, 21(7):2697-2714, 2007.

[3] D.D. Klerk, D.J. Rixen, and S.N. Voormeeren. General Framework for Dynamic Substructuring: History, Review and Classification of Techniques. AIAA Journal, 46(5):1169-1181, may 2008.

[4] A.T. Moorhouse, A.S. Elliott, and T.A. Evans. In situ measurement of the blocked force of structure-borne sound sources. Journal of Sound and Vibration, 325(4-5):679-685, sep 2009.

[5] International Organization for Standardization. BS 6897-5 1995, ISO 7626-5 1994 Experimental determination of mechanical mobility - Part 5: Measurement using impact excitation with an exciter which is not attached to the structure, 1994.

[6] J.S. Bendat and A.G. Piersol. Random Data: Analysis and Measurement Procedures. 2011.

[7] D. De Klerk. How Bias Errors Affect Experimental Dynamic Substructuring. In IMAC XVIII, pages 1101-1112, 2010.

[8] S. N. Voormeeren, D. de Klerk, and D. J. Rixen. Uncertainty quantification in experimental frequency based substructuring. Mechanical Systems and Signal Processing, 24(1):106-118, 2010.

[9] B. Cauberghe, P. Guillaume, B. Dierckx, and P. Verboven. Identification of modal parameters from inconsistent data. In IMAC XX, pages 809-815, 2002 .

[10] D.J. Rixen. How measurement inaccuracies induce spurious peaks in frequency based substructuring. In IMAC XVI, 2008.

[11] P. Gajdatsy, P. Sas, W. Desmet, K. Janssens, and H. Van da Auweraer. Effect of systematic FRF errors on matrix inversion based vibro-acoustic analysis methods. In Sensors, Instrumentation and Special Topics, Conference Proceedings of the Society for Experimental Mechanics Series, volume 6, pages 197-206, 2011. 
[12] J.S. Bendat. Statistical errors in measurement of coherence functions and input/output quantities. Journal of Sound and Vibration, 59(3):405-421, 1978.

[13] J.S. Bendat and A.G. Piersol. Engineering Applications of Correlation and Spectral Analysis. John Wiley \& Sons, Inc., 2nd edition, 1993.

[14] G.H. James, D.C. Zimmerman, and R.L. Mayes. An experimental study of frequency response function (FRF) based damage assessment tools. In IMAC-XVI, pages $151-157,1998$

[15] K. Worden. Confidence Bounds for Frequency Response Functions From Time Series Models. Mechanical Systems and Signal Processing, 12(4):559-569, 1998.

[16] H.S. Kim and T.L. Schmitz. Bivariate uncertainty analysis for impact testing. Measurement Science and Technology, 18(11):3565-3571, 2007.

[17] N.M. Ridler and M.J. Slater. An approach to the treatment of uncertainty in complex -parameter measurements. Metrologia, 39(3):295-302, 2002

[18] B.D. Hall. On the propagation of uncertainty in complex-valued quantities. Metrologia, 41(3):173-177, 2004.

[19] T. Schultz, M. Sheplak, and L.N. Cattafesta. Application of multivariate uncertainty analysis to frequency response function estimates. Journal of Sound and Vibration, 305(1-2):116-133, 2007.

[20] D. de Klerk and R. Visser. Characterization of Measurement Errors in Experimental Frequency Based Substructuring. ISMA 2010 including USD2010, pages 1881-1890, 2010.

[21] Z. Mao and M. Todd. Statistical modeling of frequency response function estimation for uncertainty quantification. Mechanical Systems and Signal Processing, 38(2):333-345, 2013.

[22] J.W.R. Meggitt, A.S. Elliott, and A.T. Moorhouse. A covariance based framework for the propagation of uncertainty through inverse problems with an application to force identification. Mechanical Systems and Signal Processing, 2018.

[23] J.R. Fonseca, M.I. Friswell, J.E. Mottershead, and A.W. Lees. Uncertainty identification by the maximum likelihood method. Journal of Sound and Vibration, 288(3):587-599, 2005.

[24] E. Zhang, J. Antoni, and P. Feissel. Bayesian force reconstruction with an uncertain model. Journal of Sound and Vibration, 331(4):798-814, 2012

[25] M.W.F. Wernsen, M.V. van der Seijs, and D. de Klerk. An indicator sensor criterion for in-situ characterisation of source vibrations. In Conference Proceedings of the Society for Experimental Mechanics, number 5. Springer, 2017

[26] F. Fahy and J. Walker. Advance Applications in Acoustics, Noise and Vibration. Spoon Press, 2004.

[27] M.V. van der Seijs, D.D. Klerk, and D.J. Rixen. General framework for transfer path analysis: History, theory and classification of techniques. Mechanical Systems and Signal Processing, pages 1-28, 2015.

[28] V. Cotoni, R.S. Langley, and M.R.F. Kidner. Numerical and experimental validation of variance prediction in the statistical energy analysis of builtup systems. Journal of Sound and Vibration, 288(3):701-728, 2005.

[29] M. Lefebvre, R. K. Keeler, R. Sobie, and J. White. Propagation of errors for matrix inversion. Nuclear Instruments and Methods in Physics Research, Section A: Accelerators, Spectrometers, Detectors and Associated Equipment, 451(2):520-528, 2000. 